\title{
Prevalence of M75 Streptococcus pyogenes Strains Harboring slaA Gene in Patients Affected by Pediatric Obstructive Sleep Apnea Syndrome in Central Italy
}

\author{
Elisa Viciani ${ }^{1,2+}$, Francesca Montagnani ${ }^{3,4 t}$, Giacinta Tordini ${ }^{3}$, Antonio Romano ${ }^{5}$, \\ Lorenzo Salerni ${ }^{5}$, Andrea De Luca ${ }^{3,4}$, Paolo Ruggiero ${ }^{1}$ and Andrea G. O. Manetti ${ }^{1 *}$ \\ ${ }^{1}$ GSK Vaccines S.r.l., Siena, Italy, ${ }^{2}$ Host-Microbiota Interaction Team, Wellcome Trust Sanger Institute, Cambridge, UK, \\ ${ }^{3}$ Department of Medical Biotechnologies, University of Siena, Siena, Italy, ${ }^{4}$ Hospital Department of Specialized and Internal \\ Medicine, University Division of Infectious Diseases, Siena, Italy, ${ }^{5}$ Clinica Otorinolaringoiatrica, Policlinico Universitario "Le \\ Scotte," Siena, Italy
}

OPEN ACCESS

Edited by:

Kate Louise Seib,

Griffith University, Australia

Reviewed by:

Vijay Pancholi,

Ohio State University at Columbus,

USA

Haider Abdul-Lateef Mousa,

University of Basrah, Iraq

*Correspondence:

Andrea G. O. Manetti

andrea.g.manetti@gsk.com

${ }^{\dagger}$ These authors have contributed equally to this work.

Specialty section:

This article was submitted to

Infectious Diseases,

a section of the journal

Frontiers in Microbiology

Received: 24 November 2016 Accepted: 13 February 2017

Published: 28 February 2017

Citation:

Viciani E, Montagnani F, Tordini G, Romano A, Salerni L, De Luca A, Ruggiero P and Manetti AGO (2017)

Prevalence of M75 Streptococcus pyogenes Strains Harboring slaA Gene in Patients Affected by Pediatric Obstructive Sleep Apnea Syndrome in Central Italy. Front. Microbiol. 8:294. doi: 10.3389/fmicb.2017.00294
Recently we reported an association between pediatric obstructive sleep apnea syndrome (OSAS) and Group A streptococcus (GAS) sub-acute chronic tonsil colonization. We showed that GAS may contribute to tonsil hyperplasia via a streptolysin O (SLO)-dependent cysteinyl leukotrienes (CysLTs) production, which can trigger T and B cell proliferation. In the present study, we characterized the GAS strains isolated from pediatric OSAS patients in comparison with a panel of age and sex matched GAS strains unrelated to OSAS, but isolated in the same area and during the same period ranging from 2009 to 2013 . We found that slaA gene, previously reported to be associated to CysLTs production pathway, was significantly associated to GAS OSAS strains. Moreover, the most numerous group (32\%) of the GAS OSAS strains belonged to M75 type, and 6 out of 7 of these strains harbored the slaA gene. Multilocus Sequence Typing (MLST) experiments demonstrated that the clone emm75/ST49/ smeZ, slaA was associated to OSAS cases. In conclusion, we found an association between slaA gene and the GAS OSAS strains, and we showed that the clone emm75/ST49 harboring genes smeZ and slaA was exclusively isolated from patients affected by OSAS, thus suggesting that this genotype might be associated to the pathogenesis of OSAS, although further studies are needed to elucidate the possible role of SlaA in tonsil hypertrophy development.

Keywords: Streptococcus pyogenes, obstructive sleep apnea syndrome (OSAS), slaA gene, Multilocus Sequence Typing (MLST), molecular epidemiology

\section{INTRODUCTION}

Obstructive sleep apnea syndrome (OSAS) has emerged in children, as the primary indication for surgical removal of adenoids and tonsils (American Thoracic Society, 1996; Marcus et al., 2012; Ramos et al., 2013). OSAS has been associated with cardiovascular, growth and neurobehavioral abnormalities, inflammation, and primarily with hypertrophy of the tonsils and adenoids (Goldbart et al., 2004; Marcus et al., 2012; Zautner, 2012; Kheirandish-Gozal et al., 2013). 
Streptococcus pyogenes is a human pathogen causing a wide range of diseases (Cunningham, 2000). Although, GAS is considered the most common single organism associated with bacterial pharyngo-tonsillitis (Sidell and Shapiro, 2012), we recently reported an association between pediatric OSAS and GAS tonsil colonization (Viciani et al., 2016). We showed that the GAS toxin SLO was involved in cysteinyl leukotrienes (CysLTs) production, through a SLO-dependent TLR4-mediated, TRIF and MyD88-dependent p38 MAPK pathway. Cysteinyl leukotrienes were able to activate primary $\mathrm{T}$ and $\mathrm{B}$ cell proliferation in vitro, which is possibly related with the development of tonsil hypertrophy, and thus with pediatric OSAS (Viciani et al., 2016).

Following the genome analysis of the M3 GAS strain MGAS315, the prophage-encoded extracellular phospholipase $\mathrm{A}_{2}$ (PLA2) SlaA was identified (Beres et al., 2002). SlaA was reported to cleave palmitic and oleic fatty acids, releasing arachidonic acid (Nagiec et al., 2004), as in the case of the cytosolic phospholipase $\mathrm{A}_{2}$ (cPLA2), which also releases the arachidonic acid from membrane phospholipids favoring its conversion to leukotrienes and CysLTs by the 5-lipoxygenase pathway (Funk, 2001). This may lead to final tonsil hypertrophy, and thus to OSAS (Viciani et al., 2016). SlaA expression is increased upon contact with cultured epithelial cells and during growth in human saliva (Banks et al., 2003; Shelburne et al., 2005); moreover, individuals with serotype M3 GAS infections produced antibodies against SlaA, indicating that the enzyme is expressed in vivo during the course of human diseases (Beres et al., 2002). Of note, the isogenic $\Delta$ slaA mutant strain was severely compromised in its ability to colonize the upper respiratory tract, leading to the conclusion that SlaA is a key colonization factor (Sitkiewicz et al., 2006).

In this study, we characterized the GAS strains isolated from pediatric OSAS patients, investigating the possible association between a panel of GAS virulence factors (including SlaA) and OSAS. Moreover, we characterized the genotype of the GAS strains isolated from OSAS patients, to investigate whether GAS tonsil colonization could be related to specific clones. To our knowledge, this is the first study that reports an association between emm75/ST49 strains harboring smeZ and slaA genes and pediatric OSAS.

\section{METHODS}

\section{Study Design, Patients, and Clinical Procedures}

As recently reported (Viciani et al., 2016), between October 2009 and December 2013, we performed a prospective case-control study on 120 pediatric patients admitted for tonsillectomy to the Otorhinolaryngology Unit of the University Hospital of Siena, which is the only reference unit for this type of surgery in the Siena province and south-east regional area (Tuscany, Central Italy). Overall population of Siena province amounts to 269,388 people among which 36,418 are pediatric subjects ( 0 to 16 years of age). The University Hospital of Siena is a 700-bed hospital and a total of 900 surgical operations/years are usually performed at the Otorhinolaryngology Unit, admitting patients from Siena city, all areas of the province and from Grosseto and Arezzo provinces. Eligible tonsillectomized patients were clinically stable children (aged $\leq 16$ years) affected by OSAS in absence of any signs and symptoms of respiratory diseases, pharyngitis and/or recurrent pharyngitis. Patients with airway obstruction and clinical features of OSAS (e.g. intermittent breathing pauses, heavy snoring, and daytime sleepiness) due to severe palatine tonsil hypertrophy (Paradise et al., 2002), were included in the OSAS group. Clinical diagnosis was confirmed by preoperative evaluation of the patients' medical histories based on specific questions to the parents/caregiver and on physical examinations. Exclusion criteria were: (I) antimicrobial treatment within 10 days before surgery; (II) coexisting chronic cardiac, hepatic, renal or pulmonary diseases; (III) acquired or congenital immunodeficiency; (IV) functional or anatomical asplenia; (V) systemic corticosteroids therapy; (VI) diabetes; (VII) craniofacial syndromes; (VIII) neuromuscular disorders, or (IX) cranial nerve palsies. Among the 120 pediatric subjects admitted for tonsillectomy at the University Hospital, 40 patients were affected by OSAS and in 19 of them we isolated one or more GAS strains, for a total of 22 isolates. In the present study, we matched them with 59 GAS strains isolated from 59 age and sex matched non-OSAS subjects recruited in the same period at the same Hospital of Siena in order to characterize the previously isolated OSAS GAS strains. All strains were community acquired because collected within $12 \mathrm{~h}$ from admission. Swabs were performed by different operators immediately before the surgery, during sedation.

\section{Isolation and Characterization of Bacterial Strains}

As previously reported (Viciani et al., 2016), tonsil swabs and cores underwent microbiological analysis. Each specimen was plated on Columbia CNA agar (CNA). Plates were incubated overnight at $37^{\circ} \mathrm{C}$ in a $5 \% \mathrm{CO}_{2}$ enriched atmosphere. Overnight cultures were identified on the basis of macro- and microscopic morphology and identity was confirmed through specific biochemical tests, according to standard methods (Murray and Masur, 2012). Glycerol stocks were made for each bacterial strain and stored at $-80^{\circ} \mathrm{C}$.

\section{Genomic DNA Extraction PCR and Sequence Analysis}

Genomic bacterial DNA employed as template for PCRs was obtained by boiling the bacterial cells, according to the CDC protocol (http://www.cdc.gov/streplab/protocol-emmtype.html). The genomic DNA was immediately used as template for PCRs or stored at $-20^{\circ} \mathrm{C}$ for subsequent studies. In order to amplify the speA, speB, speC, slo, ssa, sil, smeZ, and slaA genes, primer pairs were designed as described previously (Jing et al., 2006).

Primers SlaAF: 5'-AGTAATAAATACTATTCTATTAGCT-3', and SlaAR: 5'-TTAACATCCTATAGAACCTACTGT-3' were used to amplify PCR products and to sequence them using an Applied Biosystems model 3,730 × 1 DNA Analyzer instrument. 


\section{Co-Culture of GAS Strains with A549 Human Lung Epithelial Cells}

A549 cells were cultured on 24-well tissue culture plates with DMEM containing $25 \mathrm{mM}$ Hepes, $0.1 \%$ glutamine, 10\% fetal bovine serum and antibiotics until they reached $80 \%$ confluence. The growth medium was removed, and the cells were washed with phosphate-buffered saline (PBS). One $\mathrm{ml}$ of fresh medium without antibiotic was added, and the cells were incubated for $2 \mathrm{~h}$. During this time, overnight cultures of GAS test strains were inoculated into $10 \mathrm{ml}$ of fresh THY medium and cultured to an $\mathrm{A}_{600}=0.3$. The bacteria were collected by centrifugation, washed once in phosphate-buffered saline, and suspended in 200 microliters of PBS. Hundred microliters of bacteria were added to the A549 cells and incubated for $1 \mathrm{~h}$ and $30 \mathrm{~min}$ at $37^{\circ} \mathrm{C}$. To test the time course of induction, one well was harvested immediately to serve as a $0 \mathrm{~h}$ control. After $1 \mathrm{~h}$ and $30 \mathrm{~min}$, samples were trypsinized to collect all the bacteria and centrifuged at 14,000 $\times$ $\mathrm{g}$ for $5 \mathrm{~min}$. Pellets were immediately frozen and stored at $-80^{\circ} \mathrm{C}$.

\section{Total RNA Isolation, cDNA Synthesis, and Real-Time qPCR}

RNA extraction and RT-PCR experiments were performed modifying what previously reported (Viciani et al., 2016). Briefly, total RNA was purified from bacterial pellets co-cultured with A549 cells and collected at time 0 and after $1 \mathrm{~h} \mathrm{30}$. We performed two $30 \mathrm{~s}$ disruptions in $1 \mathrm{ml}$ TRIzol (Invitrogen), using Lysing Matrix B (MP Biomedicals; Solon, $\mathrm{OH}$ ) in a Fast Prep FP210 Homogenizer (MP Biomedical) with speed setting 6.5. Samples were incubated on ice between each disruption. After $5 \mathrm{~min}$ incubation at rt (room temperature), we centrifuged samples at $14,000 \times \mathrm{g}$ for $1 \mathrm{~min}$. Total RNA in TRIzol was isolated with Direct-zol RNA miniprep (Zymo research) according to the manufacturer's recommendations, treated with $20 \mathrm{U}$ of Turbo DNase (Ambion), and incubated at $37^{\circ} \mathrm{C}$ in a thermal bath for $30 \mathrm{~min}$. Samples were then purified and concentrated with RNA clean \& concentrator-5 (Zymo research). We estimated RNA concentration with the ND-1000 Spectrophotometer (NanoDrop Technologies, Wilmington, DE, USA). We designed primers for slaA amplification (forward: $5^{\prime}$ SlaART_F: TTTAAAGCTAGTTGGCCTGTCC- $3^{\prime}$ and reverse: SlaART_R: 5'- ACAATTAGCACCAATACCGGC-3') from S. pyogenes M3, strain GAS315 slaA sequence in GenBank (http:// www.ncbi.nlm.nih.gov/; NC_004587.1; Gene ID: 1257910) using the software Primer3 (http://simgene.com/Primer3). Previously validated reference gyrA gene expression (primers: forward: gyrART_F: 5'-CGACTTGTCTGAACGCCAAA-3' and reverse gyrART_R: 5'-TTATCACGTTCCAAACCAGTCAA-3') was used as the endogenous control for normalization of the data. Amplification efficiency was established for each of the genes from serial dilutions of $S$. pyogenes genomic DNA. The reactions were performed in a Light Cycler 480 II (Roche) using the Light Cycler RNA amplification kit SYBR green I (Roche) according to the manufacturer's instructions. Each gene was analyzed in triplicate and results were evaluated using Light Cycler ${ }^{\circledR} 480 \mathrm{SW}$ 1.5 software (Roche). All reactions amplified a single product as determined by melting curve analysis. The expression of
slaA gene as compared with the reference gene gyrA expression was evaluated with the relative quantification method $(\Delta \Delta \mathrm{CT}$ Method). Data were represented as relative amounts of mRNA normalized to a gyrA control.

\section{Antibiotic Susceptibility Tests}

In vitro susceptibility to macrolides and lincosamides was tested in all 81 isolates. Assays were performed and the results were interpreted according to Clinical Laboratory Standards Institute (CLSI) indications (CLSI, 2012). Susceptibility to 14- and 16membered ring macrolides (erythromycin, spiramycin) and to lincosamides (clindamycin) was determined by the Kirby-Bauer disk diffusion method on Mueller-Hinton agar with 5\% sheep blood plates (Oxoid/Thermo Scientific). In addition to the CLSI double disk indication, a triple-disk diffusion test was performed to highlight any possible heterogeneity of inducible resistance, as described previously (Giovanetti et al., 1999).

\section{emm Typing}

emm typing was carried out according to CDC guidelines (http:// www.cdc.gov/streplab/protocol-emm-type.html). Enzymatic extraction of streptococcal genomic DNA was replaced by bacterial boiling in distilled water (Tewodros and Kronvall, 2005) and PCR amplification of the emm gene was carried out by the CDC protocol and PCR product sequencing. Sequenced PCR products were then compared with the $\mathrm{CDC}$ database to retrieve the emm type.

\section{MLST Analysis}

Multilocus Sequence Typing (MLST) analysis was conducted on all the M75 strains, as previously described (Enright et al., 2001). This procedure was performed by sequencing seven housekeeping genes ( $g k i, g t r, m u r I, m u t S$, recP, xpt, and yqiL) according to the $S$. pyogenes MLST website (http://pubmlst.org/ spyogenes/) sited at the University of Oxford (Jolley and Maiden, 2010). We then compared M75 GAS OSAS strains with M75 GAS strains belonging to three different collections. Collection one contained a miscellaneous of M75 strains isolated from patients affected by a number of different pathologies, and was obtained from the University of Siena, Italy $(n=5)$; collection two contained strains isolated from patients affected by throat or skin infection, and was obtained from the University of Rostock, Germany $(n=7)$; collection three contained strains isolated from patients affected exclusively by pharyngitis, and was obtained from Baylor College of Medicine Houston, Texas, USA $(n=16)$.

\section{Statistics}

Non-parametric data are presented as median. Data were analyzed using Z-test (Table 1 sex data; Tables 2-4), nonparametric Kolmogorov-Smirnov (Table 1 age data), or unpaired $t$-test (Figure 1). For all analyses, $p<0.05$ was considered significant. Analyses were done with R software and GraphPad Prism 7.02 software.

\section{Ethical Declarations}

This study was carried out in accordance with the recommendations of the Ethical Committee of the Azienda Ospedaliera Universitaria Senese at Siena Hospital with written 
informed consent from all subjects. All subjects and in particular all the next of kin, caretakers, or guardians on the behalf of the minors/children participants gave written informed consent in accordance with the Declaration of Helsinki. The protocol was approved by the Ethical Committee of the Azienda Ospedaliera Universitaria Senese at Siena Hospital.

\section{RESULTS}

\section{Presence of slaA Gene Is Prevalent in Pediatric OSAS Patients}

During the characterization of the GAS strains isolated from pediatric ( $\leq 16$ years) OSAS patients (Viciani et al., 2016), we amplified by PCR eight genes encoding virulence factors (Jing et al., 2006) both in the 22 GAS strains isolated from 19 OSAS patients and in 59 GAS strains isolated from 59 age and sex matched non-OSAS subjects recruited in the same period and at the same Hospital of Siena. No significant differences were noted in the demographics of the matched populations (Table 1). As shown in Table 2, speB, and slo were detected in all the GAS isolates analyzed, confirming to be speciesspecific, whereas the ssa superantigen was not present in OSAS or in non-OSAS isolates. Interestingly, slaA gene was found significantly associated $(p=0.002)$ with GAS strains isolated from OSAS patients as compared with GAS strains isolated from non-OSAS subjects, suggesting a possible role of this virulence factor in the development of tonsil hypertrophy. None of the other genes analyzed were found to occur significantly more frequently in the GAS OSAS group vs. the non-OSAS group. No significant difference was found in macrolides and lincosamides

TABLE 1 | Demographics of the study population stratified by OSAS (obstructive sleep apnoea syndrome), and matched with non-OSAS subjects.

\begin{tabular}{lccc}
\hline Characteristics & OSAS & Non-OSAS & $\boldsymbol{p}$-value \\
\hline No. of patients & 19 & 59 & - \\
Age, years & $6(5-9)(4-11)$ & $6(5-9)(1-11)$ & 0.77 \\
Sex, male & $10(52.6 \%)$ & $31(52.5 \%)$ & 0.99
\end{tabular}

Age, median [interquartile range (IQR)] and (minimum age - maximum age). Sex, male numbers (\%). Statistical analysis was performed using a non-parametric KolmogorovSmirnov test on the distribution of age data, and a Z-test on the proportion of cases and controls for sex data (significance level $p=0.05$ ). chemo-susceptibility patterns of the isolates from OSAS and non-OSAS subjects (Table 3 ).

\section{Presence of slaA Gene Correlates with M75 Type}

The emm type analysis of the GAS strains isolated from OSAS cases or from non-OSAS subjects showed that most of the isolates $(32 \%, n=7)$ from the GAS OSAS group belonged to M75 type, whereas only 4 out of $59(6.8 \%)$ of the GAS strains isolated from non-OSAS subjects belonged to this $\mathrm{M}$ type $(p=0.03)$. Moreover, no other $\mathrm{M}$ type was found to be prevalent in OSAS or non-OSAS group (Table 4). Interestingly, 6 out of the 7 M75 strains isolated from patients affected by OSAS harbored the slaA gene (Table S1) which, when sequenced, did not presented any allelic variation (data not shown). Moreover, when all 6 M75 stains were co-cultured with A549 human epithelial lung cells, they increased slaA gene expression levels (Figure 1), showing to harbor host epithelial cell-inducible expression of slaA gene. We then investigated the presence of slaA gene in M75 non-OSAS GAS strains isolated from patients affected by pharyngo-tonsillitis, arthropathy, arthralgia, otitis, skin infection or superinfection during infectious mononucleosis, belonging to three different collections of GAS strains isolated in Italy $(n=$ $5)$, Germany $(n=7)$ and USA $(n=16)$. As shown in Table 5 , we obtained four different patterns. Pattern one, harboring genes

TABLE 3 | Macrolides and lincosamides antimicrobial susceptibility of GAS isolates from OSAS patients and from matched non-OSAS subjects.

\begin{tabular}{lccc}
\hline & Erythro & Clinda & Spira \\
\hline GAS OSAS (22) & & & \\
Susceptible & $95.5 \%(21)$ & $95.5 \%(21)$ & $95.5 \%(21)$ \\
Intermediate & $0.0 \%$ & $0.0 \%$ & $0.0 \%$ \\
Resistant & $4.5 \%(1)$ & $4.5 \%(1)$ & $4.5 \%(1)$ \\
GAS NON-OSAS (59) & & & \\
Susceptible & $93.2 \%(55)$ & $94.9 \%(56)$ & $94.9 \%(56)$ \\
Intermediate & $0.0 \%$ & $0.0 \%$ & $0.0 \%$ \\
Resistant & $6.8 \%(4)$ & $5.1 \%(3)$ & $5.1 \%(3)$ \\
$p$ & 0.71 & 0.92 & 0.92 \\
\hline
\end{tabular}

Antibiotics, erythromycin (Erythro), clindamycin (Clinda) and Spiramycin (Spira). Statistical analysis was performed using a Z-test on the proportion of Susceptible and Intermediate/Resistant strains (significance level $p=0.05$ ). The percent of isolates is reported in each cell, along with the corresponding number of isolates in brackets, when appropriate.

TABLE 2 | Distribution of virulence genes among GAS strains isolated from pediatric OSAS patients and matched GAS strains isolated from non-OSAS subjects.

\begin{tabular}{|c|c|c|c|c|c|c|c|c|}
\hline & spe $A$ & speB & speC & smeZ & slo & ssa & sil & slaA \\
\hline GAS OSAS (22) & $18.1 \%(4)$ & $100.0 \%$ & $45.5 \%(10)$ & $72.7 \%(16)$ & $100.0 \%$ & $0.0 \%$ & $18.2 \%(4)$ & $45.5 \%(10)$ \\
\hline GAS non-OSAS (59) & $25.4 \%(15)$ & $100.0 \%$ & $45.7 \%(27)$ & $74.6 \%(44)$ & $100.0 \%$ & $0.0 \%$ & $18.6 \%(11)$ & $13.5 \%(8)$ \\
\hline$p$ & 0.49 & 1 & 0.98 & 0.86 & 1 & 1 & 0.96 & 0.002 \\
\hline
\end{tabular}

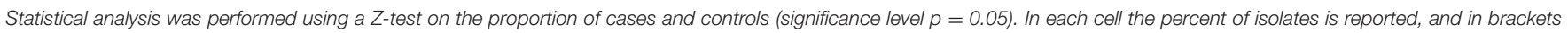
the corresponding number of isolates when appropriate. 
TABLE 4 | Distribution of emm types among GAS strains isolated from OSAS patients and from matched non-OSAS subjects.

\begin{tabular}{lccc}
\hline emm type & OSAS $\boldsymbol{n}$. (\%) & Non-OSAS $\boldsymbol{n}$. (\%) & p-value \\
\hline emm75 & $7(31.8)$ & $4(6.8)$ & 0.003 \\
emm4 & $3(13.6)$ & $4(6.8)$ & 0.32 \\
emm89 & $3(13.6)$ & $10(16.9)$ & 0.36 \\
emm3 & $2(9.1)$ & $1(1.7)$ & 0.11 \\
emm12 & $2(9.1)$ & $8(13.5)$ & 0.58 \\
emm1 & $1(4.5)$ & $7(11.8)$ & 0.32 \\
emm5 & $1(4.5)$ & $4(6.8)$ & 0.71 \\
emm28 & $1(4.5)$ & $3(5.1)$ & 0.92 \\
emm77 & $1(4.5)$ & $1(1.7)$ & 0.45 \\
emm87 & $1(4.5)$ & $3(1.7)$ & 0.45 \\
emm18 & $0(0)$ & $3(5.1)$ & 0.28 \\
emm6 & $0(0)$ & $2(3.4)$ & 0.28 \\
emm11 & $0(0)$ & $1(1.7)$ & 0.38 \\
emm78 & $0(0)$ & $3(5.1)$ & 0.54 \\
emm29 & $0(0)$ & $2(3.4)$ & 3.28 \\
emm61 & $0(0)$ & $1(1.7)$ & 0.38 \\
emm92 & $0(0)$ & $1(1.7)$ & 0.54 \\
emm9 & $0(0)$ & $100.0)$ & 0.54 \\
Total & $(100.0)$ & & - \\
\hline
\end{tabular}

Statistical analysis was performed using a Z-test on the proportion of cases and controls (significance level $p=0.05$ ).

smeZ and slaA, was prevalent in strains isolated from OSAS patients in Italy. Pattern two, harboring genes $s p e A$, smeZ, and slaA, was prevalent in strains isolated from patients affected by different pathologies in Italy. Pattern three, harboring gene smeZ was prevalent in strains isolated from patients affected by throat infection in Germany. Pattern four, harboring genes speC and smeZ was prevalent in strains isolated from patients affected by pharyngitis in USA. The genes speB and slo were not reported as they are species-specific. Interestingly, only 4 out of the 28 nonOSAS GAS strains harbored the slaA gene (Table 5). Therefore, our results indicated a significant association $(p=0.00018)$ of slaA gene with GAS M75 strains isolated from patients affected by OSAS.

\section{No Correlation was Found between ST and OSAS Cases}

Based on this finding, we decided to perform an MLST analysis of M75 strains, in order to investigate whether the pattern harboring slaA gene was due to clonality or to different GAS strains. Overall, emm75/ST49 type was isolated in Italy and USA, harboring mostly pattern one, two and four, but also in one case pattern three (Table 5). On the other hand, clone emm75/ST150 was prevalent in pattern three in GAS strains isolated in Germany, harboring neither slaA gene nor speC gene. However, emm75/ST150 strains were also found harboring pattern one and pattern three (Table 5). As shown in Table 5, the MLST analysis revealed that a single clone was responsible for pattern one in M75 strains isolated from OSAS patients. In fact, the clone emm75/ST49 harboring slaA and smeZ genes

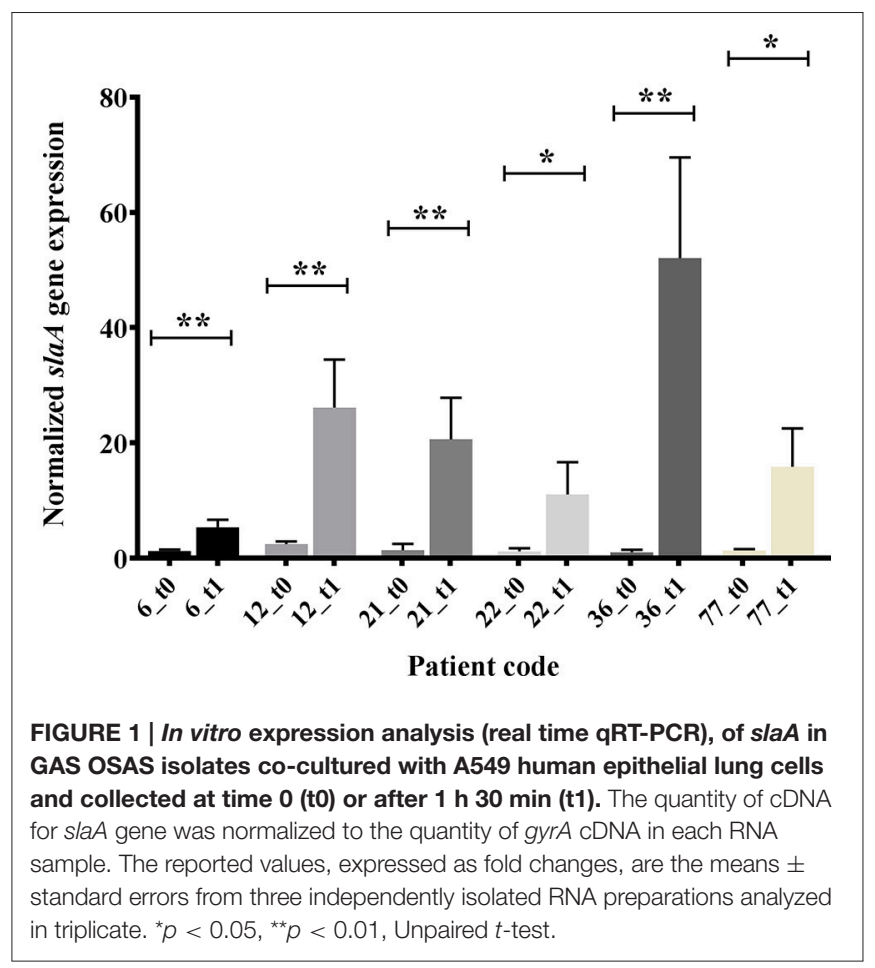

was associated to OSAS in a period ranging from 2009 to 2011 (Table S1); however, when speA gene was additionally present (pattern two) no association with OSAS patients was observed.

\section{The Genotype M75/ST49 Harboring slaA Gene Is Prevalent in OSAS Cases}

The characterization of the GAS strains isolated from OSAS patients showed the significant prevalence of slaA gene in these isolates as compared with non-OSAS GAS strains isolated in the same area. Furthermore, 6 out of $7(85.7 \%)$ of the M75 GAS strains isolated from OSAS patients harbored slaA gene, whereas the analysis for the presence of this gene in a panel of non-OSAS M75 strains, isolated in different countries, showed that only 4 out of 28 strains (14.3\%) harbored slaA gene. Finally, we showed that all the M75/ST49 GAS strains colonizing OSAS patients harbored genes sme $Z$ and slaA, a unique clone circulating in a period ranging from 2009 to 2011 . In fact, interestingly, this GAS clone was never isolated from non-OSAS patients examined in the same area and in the same period. Of note, unlike slaA, the superantigene smeZ was not prevalently associated with GAS strains isolated from OSAS patients when compared with GAS strains isolate from non-OSAS subjects $(p=0.86$, Table 2), suggesting that this virulence factor is not particularly associated to patients affected by OSAS. It has also to be noted that in M75/ST49 strains the presence of speC gene is negatively associated to OSAS cases $(p=0.0003)$.

Taken together these data show the association of GAS clone emm75/ST49 harboring slaA gene with pediatric patients affected by OSAS, leading to hypothesize a possible role of the SlaA protein in the pathogenesis of tonsil hypertrophy, and thus possibly of obstructive sleep apnea syndrome. 


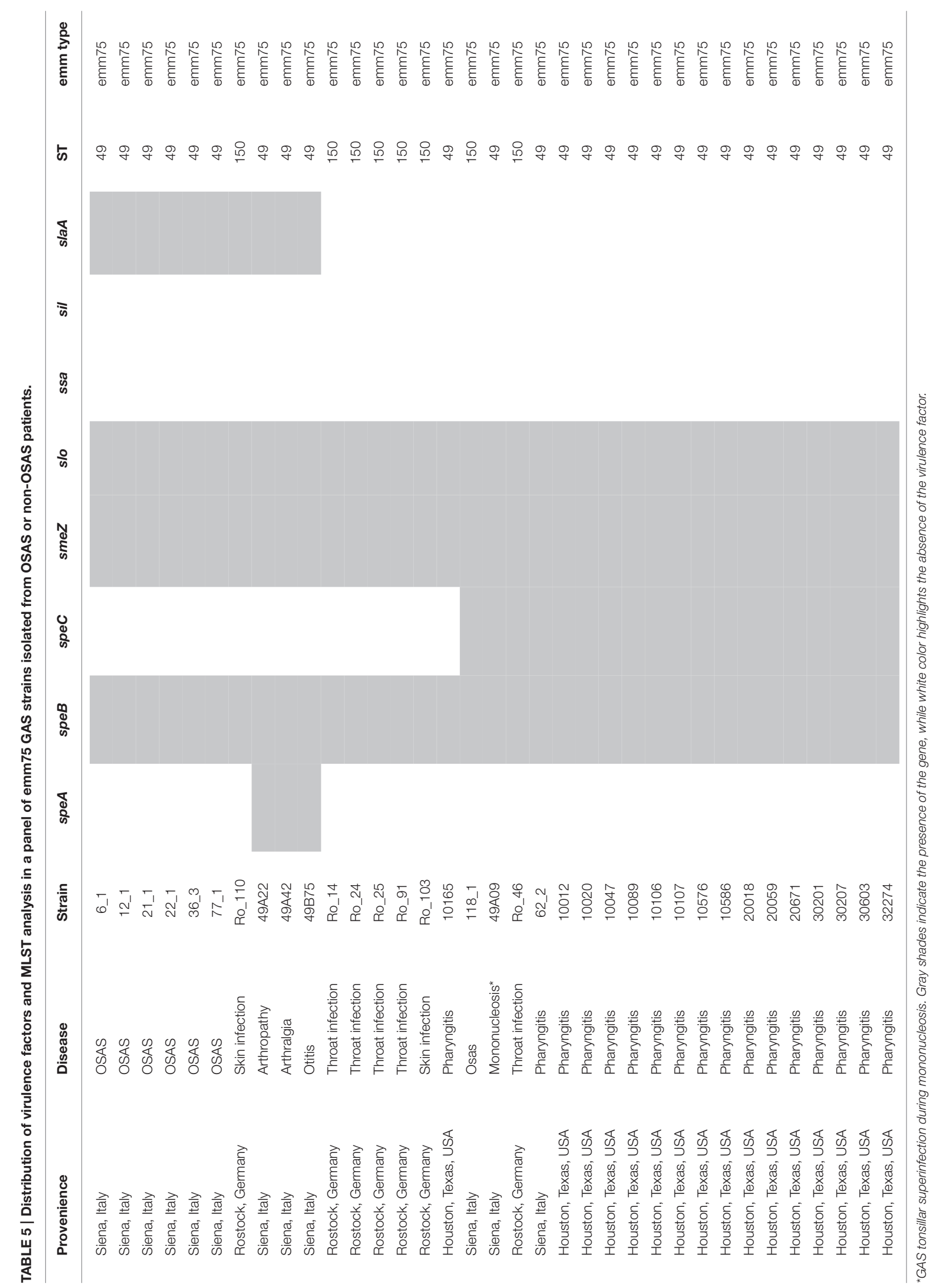




\section{DISCUSSION}

We recently reported an association between pediatric OSAS and GAS tonsil colonization (Viciani et al., 2016). Based on our data, we hypothesized a mechanism of pathogenesis where tonsil hypertrophy, the main risk factor for pediatric OSAS, could be determined by the proliferative effect that the GAS toxin SLOdependent TLR4-mediated, TRIF and MyD88-dependent CysLTs production has on tonsil T and B cells (Viciani et al., 2016). Here, we found that slaA gene, previously reported to be involved in leukotrienes production pathway (Funk, 2001), is significantly associated to GAS OSAS strains and in particular to the clone emm75/ST49 harboring smeZ and slaA genes.

In 1997, erythromycin resistance was reported to be in $53 \%$ of GAS isolates from Siena; 10 years later the same resistance phenotype decreased to $16 \%$, these phenotypes being equally distributed between $M$ (14 and 15 ring member macrolide-resistant) and MLS (all macrolide, lincosamide, and streptogramin-resistant) (Cresti et al., 2002; Montagnani et al., 2009). Although, the genotype emm $75 /$ ST 49 was one of the most frequent $\mathrm{M}$-phenotype, carrying $\operatorname{mef}(A)$ gene, isolated during surveys conducted in USA, Spain, Korea and Japan (Green et al., 2006; Ardanuy et al., 2010; Takahashi et al., 2016), in our study we found 1 MLS resistant phenotype in all the GAS strains isolated from the OSAS patients, and 3 MLS resistant phenotypes plus $1 \mathrm{M}$ phenotype in strains isolated from matched non-OSAS group, no one belonging to M75 type. This very low macrolide resistance rate was further confirmed by a recent study conducted in the same area (Olivieri et al., 2015). In a study conducted in Portugal between 2,000 and 2,005, the genotype emm75/ST150 was significantly associated with pharyngitis (Friaes et al., 2012); however, interestingly, a mucoid form of a GAS strain M75/ST49 has recently been reported to cause a severe acute otitis media (Kakuta et al., 2014).

The analysis of the presence of eight virulence factors in the genome of the GAS OSAS strains showed the species-specificity of speB and slo as previously reported (Jing et al., 2006; LucaHarari et al., 2009). Moreover, the superantigen gene ssa was not found in our isolates, but was present in $31 \%$ of the strains isolated in a European survey (Luca-Harari et al., 2009), and in $23 \%$ of the isolates in a Chinese study (Jing et al., 2006). The superantigen gene sme $Z$ was highly represented in our isolates, as described in other surveys (Jing et al., 2006; LucaHarari et al., 2009). In agreement with previous data, speA was associated with M1 and M3, often involved in severe infections with high mortality, whereas speC was isolated in several other types, also associated with high mortality (Schmitz et al., 2003; Jing et al., 2006; Luca-Harari et al., 2009). The M1 and M3 strains have the gene speA and lack gene speC, suggesting that the presence of speA affects the acquisition of speC (Schmitz et al., 2003). In China speA was more prevalent in invasive than in epipharynx isolates, while speC was significantly overrepresented in epipharynx isolates (Jing et al., 2006). Another gene related to invasive GAS strains is sil (Hidalgo-Grass et al., 2004), which we found in $18.2 \%$ of OSAS strains similarly to GAS isolated from China (Jing et al., 2006).

Following the genome analysis of the M3 GAS strain MGAS315, a prophage-encoded extracellular phospholipase
$\mathrm{A}_{2}$ (PLA2) named SlaA involved in the production of pro-inflammatory lipid mediators such as leukotrienes was identified. SlaA had not been present in M3 GAS strains before 1987, when a resurgence of severe invasive disease episodes occurred (Beres et al., 2002; Ikebe et al., 2002). These data, together with the reported lack of allelic variation, suggest that the sla $A$ gene was recently acquired by transduction by a distinct serotype M3 sub-clone that then spread globally (Nagiec et al., 2004; Sitkiewicz et al., 2007). It has been reported that slaA was present, even if uncommonly, in few other serotypes, including M1, M2, M4, M6, M22, M28, and M75 strains (Nagiec et al., 2004).

Of note, slaA gene was found in $45.5 \%$ of the GAS strains isolated from OSAS patients, whereas the occurrence rate of this gene has been reported to be much lower (10.8\% in Europe, USA or Canada and $4.65 \%$ in China) (Nagiec et al., 2004; Jing et al., 2006). On this regard, GAS M75 strains harboring slaA gene, isolated in $85.7 \%$ of the OSAS subjects colonized by GAS, were reported to be uncommon (Nagiec et al., 2004; Jing et al., 2006; Kittang et al., 2011) and, when occurring, associated with invasive strains (Nagiec et al., 2004). Recently, it has been described a high expression of sla $A$ and of the adjacent gene speK in a skin-tropic invasive GAS strain, implying an involvement of SlaA in hostpathogen interactions in the skin and a possible role in enhancing virulence and fitness in adaption to host niches (Bao et al., 2016).

It has been shown that slaA expression was induced upon contact with cultured human pharyngeal epithelial cells (confirmed in this study) and during growth in human saliva (Banks et al., 2003; Shelburne et al., 2005), and that individuals with serotype M3 GAS infections seroconvert to SlaA, indicating that the enzyme is expressed in vivo during the course of human diseases (Beres et al., 2002). Moreover, interestingly, in a cynomolgus macaque model of pharyngitis (Sumby et al., 2005; Virtaneva et al., 2005), the isogenic slaA mutant strain was severely compromised in its ability to colonize the upper respiratory tract, leading to the conclusion that SlaA is a key colonization factor (Sitkiewicz et al., 2007). It has been demonstrated that SlaA, which is involved in the generation of pro-inflammatory lipid mediators such as leukotrienes (Nagiec et al., 2004), is regulated by TLR4 signaling in LPSactivated macrophages and that the regulation occurs through TLR4-mediated MyD88- and TRIF-dependent MAPK signaling pathways (Qi and Shelhamer, 2005). In this study, we observed a significant association between OSAS cases and GAS strains genotype emm75/ST49 harboring slaA,. Given that SlaA can induce CysLTs production and that the latter are involved in tonsillar T and B cell proliferation, these results could enforce the recent discovery of a possible role of GAS virulence factors in tonsil hypertrophy and pediatric OSAS development (Viciani et al., 2016). However the found correlation does not imply causation; the multifactorial nature of the disease and the fact that our data refer to a limited number of cases and are from a single center in central Italy suggest that the study sample might not be representative of the whole Italian population. Ideally our studies should be replicated in a large independent, multicenter cohort. Moreover, since only a subset $(45.5 \%)$ of GAS OSAS strains in our study harbors slaA gene, we cannot rule out the importance of other risk factors, such as SLO 
toxin; thus slaA gene should be considered as a part of a combination of factors, which may have a possible role in chronic diseases.

\section{AUTHOR CONTRIBUTIONS}

Conceived and designed the experiments, reviewed and approved the final version of the manuscript and analyzed the data: EV, FM, GT, AR, LS, AD, PR, and AM. Performed the experiments: EV, GT, and AM. Wrote the paper: EV, FM, PR, and AM.

\section{REFERENCES}

American Thoracic Society (1996). Standards and indications for cardiopulmonary sleep studies in children. Am. J. Respir. Crit. Care Med. 153, 866-878.

Ardanuy, C., Domenech, A., Rolo, D., Calatayud, L., Tubau, F., Ayats, J., et al. (2010). Molecular characterization of macrolide- and multidrugresistant Streptococcus pyogenes isolated from adult patients in Barcelona, Spain (1993-2008). J. Antimicrob. Chemother. 65, 634-643. doi: 10.1093/jac/ dkq006

Banks, D. J., Lei, B., and Musser, J. M. (2003). Prophage induction and expression of prophage-encoded virulence factors in group a Streptococcus serotype M3 strain MGAS315. Infect Immun 71, 7079-7086. doi: 10.1128/IAI.71.12.7079-7086.2003

Bao, Y. J., Liang, Z., Mayfield, J. A., Donahue, D. L., Carothers, K. E., Lee, S. W., et al. (2016). Genomic characterization of a pattern D Streptococcus pyogenes emm53 isolate reveals a genetic rationale for invasive skin tropicity. J. Bacteriol. 198, 1712-1724. doi: 10.1128/JB.01019-15

Beres, S. B., Sylva, G. L., Barbian, K. D., Lei, B., Hoff, J. S., Mammarella, N. D., et al. (2002). Genome sequence of a serotype M3 strain of group a Streptococcus: phage-encoded toxins, the high-virulence phenotype, and clone emergence. Proc. Natl. Acad. Sci. U.S.A. 99, 10078-10083. doi: 10.1073/pnas.1522 98499

CLSI (2012). Performance Standards for Antimicrobial Susceptibility Testings; 22nd Informational Supplement M100-S22. Wayne, PA: Clinical, Laboratory Standards Institute.

Cresti, S., Lattanzi, M., Zanchi, A., Montagnani, F., Pollini, S., Cellesi, C., et al. (2002). Resistance determinants and clonal diversity in group A streptococci collected during a period of increasing macrolide resistance. Antimicrob. Agents Chemother. 46, 1816-1822. doi: 10.1128/AAC.46.6.1816-1822.2002

Cunningham, M. W. (2000). Pathogenesis of group a streptococcal infections. Clin. Microbiol. Rev. 13, 470-511. doi: 10.1128/CMR.13.3.470-511.2000

Enright, M. C., Spratt, B. G., Kalia, A., Cross, J. H., and Bessen, D. E. (2001). Multilocus sequence typing of Streptococcus pyogenes and the relationships between emm type and clone. Infect. Immun. 69, 2416-2427. doi: 10.1128/IAI.69.4.2416-2427.2001

Friaes, A., Pinto, F. R., Silva-Costa, C., Ramirez, M., Melo-Cristino, J., and Portuguese Group for the Study of Streptococcal, I. (2012). Group A streptococci clones associated with invasive infections and pharyngitis in Portugal present differences in emm types, superantigen gene content and antimicrobial resistance. BMC Microbiol. 12:280. doi: 10.1186/1471-2180-12-280

Funk, C. D. (2001). Prostaglandins and leukotrienes: advances in eicosanoid biology. Science 294, 1871-1875. doi: 10.1126/science.294.5548.1871

Giovanetti, E., Montanari, M. P., Mingoia, M., and Varaldo, P. E. (1999). Phenotypes and genotypes of erythromycin-resistant Streptococcus pyogenes strains in Italy and heterogeneity of inducibly resistant strains. Antimicrob. Agents Chemother. 43, 1935-1940.

Goldbart, A. D., Goldman, J. L., Li, R. C., Brittian, K. R., Tauman, R., and Gozal, D. (2004). Differential expression of cysteinyl leukotriene receptors 1 and 2 in tonsils of children with obstructive sleep apnea syndrome or recurrent infection. Chest 126, 13-18. doi: 10.1378/chest.126.1.13

Green, M. D., Beall, B., Marcon, M. J., Allen, C. H., Bradley, J. S., Dashefsky, B., et al. (2006). Multicentre surveillance of the prevalence and molecular epidemiology

\section{ACKNOWLEDGMENTS}

The authors thank Dr. Cesare Biagini for tonsil surgery and Prof. Walter Livi, for helpful discussions.

\section{SUPPLEMENTARY MATERIAL}

The Supplementary Material for this article can be found online at: http://journal.frontiersin.org/article/10.3389/fmicb. 2017.00294/full\#supplementary-material

of macrolide resistance among pharyngeal isolates of group A streptococci in the USA. J. Antimicrob. Chemother. 57, 1240-1243. doi: 10.1093/jac/dkl101

Hidalgo-Grass, C., Dan-Goor, M., Maly, A., Eran, Y., Kwinn, L. A., Nizet, V., et al. (2004). Effect of a bacterial pheromone peptide on host chemokine degradation in group A streptococcal necrotising soft-tissue infections. Lancet 363, 696-703. doi: 10.1016/S0140-6736(04)15643-2

Ikebe, T., Wada, A., Inagaki, Y., Sugama, K., Suzuki, R., Tanaka, D., et al. (2002). Dissemination of the phage-associated novel superantigen gene speL in recent invasive and noninvasive Streptococcus pyogenes M3/T3 isolates in Japan. Infect. Immun. 70, 3227-3233. doi: 10.1128/IAI.70.6.3227-3233.2002

Jing, H. B., Ning, B. A., Hao, H. J., Zheng, Y. L., Chang, D., Jiang, W., et al. (2006). Epidemiological analysis of group A streptococci recovered from patients in China. J. Med. Microbiol. 55, 1101-1107. doi: 10.1099/jmm.0.46243-0

Jolley, K. A., and Maiden, M. C. (2010). BIGSdb: scalable analysis of bacterial genome variation at the population level. BMC Bioinformatics 11:595. doi: 10.1186/1471-2105-11-595

Kakuta, R., Yano, H., Hidaka, H., Miyazaki, H., Irimada, M., Oda, K., et al. (2014). Severe acute otitis media caused by mucoid Streptococcus pyogenes in a previously healthy adult. Tohoku J. Exp. Med. 232, 301-304. doi: $10.1620 /$ tjem.232.301

Kheirandish-Gozal, L., Kim, J., Goldbart, A. D., and Gozal, D. (2013). Novel pharmacological approaches for treatment of obstructive sleep apnea in children. Expert Opin. Investig. Drugs 22, 71-85. doi: 10.1517/13543784.2013.735230

Kittang, B. R., Skrede, S., Langeland, N., Haanshuus, C. G., and Mylvaganam, H. (2011). emm gene diversity, superantigen gene profiles and presence of SlaA among clinical isolates of group A, C and G streptococci from western Norway. Eur. J. Clin. Microbiol. Infect. Dis. 30, 423-433. doi: 10.1007/s10096-0101105-X

Luca-Harari, B., Darenberg, J., Neal, S., Siljander, T., Strakova, L., Tanna, A., et al. (2009). Clinical and microbiological characteristics of severe Streptococcus pyogenes disease in Europe. J. Clin. Microbiol. 47, 1155-1165. doi: 10.1128/JCM.02155-08

Marcus, C. L., Brooks, L. J., Draper, K. A., Gozal, D., Halbower, A. C., Jones, J., et al. (2012). Diagnosis and management of childhood obstructive sleep apnea syndrome. Pediatrics 130, 576-584. doi: 10.1542/peds.2012-1671

Montagnani, F., Stolzuoli, L., Croci, L., Rizzuti, C., Arena, F., Zanchi, A., et al. (2009). Erythromycin resistance in Streptococcus pyogenes and macrolide consumption in a central Italian region. Infection 37, 353-357. doi: 10.1007/s15010-008-8023-1

Murray, P. R., and Masur, H. (2012). Current approaches to the diagnosis of bacterial and fungal bloodstream infections in the intensive care unit. Crit. Care Med. 40, 3277-3282. doi: 10.1097/CCM.0b013e318270e771

Nagiec, M. J., Lei, B., Parker, S. K., Vasil, M. L., Matsumoto, M., Ireland, R. M., et al. (2004). Analysis of a novel prophage-encoded group A Streptococcus extracellular phospholipase A(2). J. Biol. Chem. 279, 45909-45918. doi: 10.1074/jbc.M405434200

Olivieri, R., Morandi, M., Zanchi, A., Tordini, G., Pozzi, G., De Luca, A., et al. (2015). Evolution of macrolide resistance in Streptococcus pyogenes over 14 years in an area of central Italy. J. Med. Microbiol. 64, 1186-1195. doi: $10.1099 / \mathrm{jmm} .0 .000146$

Paradise, J. L., Bluestone, C. D., Colborn, D. K., Bernard, B. S., Rockette, H. E., and Kurs-Lasky, M. (2002). Tonsillectomy and Adenotonsillectomy for 
recurrent throat infection in moderately affected children. Pediatrics 110, 7-15. doi: 10.1542 peds.110.1.7

Qi, H. Y., and Shelhamer, J. H. (2005). Toll-like receptor 4 signaling regulates cytosolic phospholipase A2 activation and lipid generation in lipopolysaccharide-stimulated macrophages. J. Biol. Chem. 280, 38969-38975. doi: 10.1074/jbc.M509352200

Ramos, S. D., Mukerji, S., and Pine, H. S. (2013). Tonsillectomy and adenoidectomy. Pediatr. Clin. North Am. 60, 793-807. doi: 10.1016/j.pcl.2013. 04.015

Schmitz, F. J., Beyer, A., Charpentier, E., Normark, B. H., Schade, M., Fluit, A. C., et al. (2003). Toxin-gene profile heterogeneity among endemic invasive European group A streptococcal isolates. J. Infect. Dis. 188, 1578-1586. doi: $10.1086 / 379230$

Shelburne, S. A. III., Granville, C., Tokuyama, M., Sitkiewicz, I., Patel, P., and Musser, J. M. (2005). Growth characteristics of and virulence factor production by group A Streptococcus during cultivation in human saliva. Infect. Immun. 73, 4723-4731. doi: 10.1128/IAI.73.8.4723-4731.2005

Sidell, D., and Shapiro, N. L. (2012). Acute tonsillitis. Infect. Disord. Drug Targets $12,271-276$

Sitkiewicz, I., Nagiec, M. J., Sumby, P., Butler, S. D., Cywes-Bentley, C., and Musser, J. M. (2006). Emergence of a bacterial clone with enhanced virulence by acquisition of a phage encoding a secreted phospholipase A2. Proc. Natl. Acad. Sci. U.S.A. 103, 16009-16014. doi: 10.1073/pnas.0607669103

Sitkiewicz, I., Stockbauer, K. E., and Musser, J. M. (2007). Secreted bacterial phospholipase A2 enzymes: better living through phospholipolysis. Trends Microbiol. 15, 63-69. doi: 10.1016/j.tim.2006.12.003

Sumby, P., Barbian, K. D., Gardner, D. J., Whitney, A. R., Welty, D. M., Long, R. D., et al. (2005). Extracellular deoxyribonuclease made by group A Streptococcus assists pathogenesis by enhancing evasion of the innate immune response. Proc. Natl. Acad. Sci. U.S.A. 102, 1679-1684. doi: 10.1073/pnas.0406641102

Takahashi, T., Arai, K., Lee, D. H., Koh, E. H., Yoshida, H., Yano, H., et al. (2016). Epidemiological study of erythromycin-resistant Streptococcus pyogenes from Korea and Japan by emm genotyping and Multilocus sequence typing. Ann. Lab Med. 36, 9-14. doi: 10.3343/alm.2016.36.1.9

Tewodros, W., and Kronvall, G. (2005). M protein gene (emm type) analysis of group A (-hemolytic streptococci from Ethiopia reveals unique patterns. J. Clin. Microbiol. 43, 4369-4376. doi: 10.1128/JCM.43.9.4369-4376.2005
Viciani, E., Montagnani, F., Tavarini, S., Tordini, G., Maccari, S., Morandi, M., et al. (2016). Paediatric obstructive sleep apnoea syndrome (OSAS) is associated with tonsil colonisation by Streptococcus pyogenes. Sci. Rep. 6:20609. doi: 10.1038/srep20609

Virtaneva, K., Porcella, S. F., Graham, M. R., Ireland, R. M., Johnson, C. A., Ricklefs, S. M., et al. (2005). Longitudinal analysis of the group A Streptococcus transcriptome in experimental pharyngitis in cynomolgus macaques. Proc. Natl. Acad. Sci. U.S.A. 102, 9014-9019. doi: 10.1073/pnas.0503 671102

Zautner, A. E. (2012). Adenotonsillar disease. Recent Pat. Inflamm. Allergy Drug Discov. 6, 121-129. doi: 10.2174/187221312800166877

Conflict of Interest Statement: AM and PR were employees of Novartis Vaccines and Diagnostics Srl at the time of the study. Following the acquisition of Novartis Vaccines by the GSK group of companies in March, 2015, AM is now employee of the GSK group of companies, while PR retired. EV was a PhD Student of the University of Siena at the time of the study and supervised by Novartis Vaccines and Diagnostics Srl. EV has now a postdoctoral position at Wellcome Trust Sanger Institute, $\mathrm{UK}$. $\mathrm{AD}$ has a personal fee from Merck Sharp and Dohme. AD, FM, $\mathrm{AR}$, and LS have done contract research for Novartis Vaccine and Diagnostics S.r.l. (now acquired by the GSK group of companies) on behalf of the Hospital University of Siena, Italy. This work was sponsored by Novartis Vaccines and Diagnostics Srl, now acquired by the GSK group of companies, who were involved in all stages of the study conduct and analysis. GSK took responsibility for all costs incurred in publishing.

The other authors declare that the research was conducted in the absence of any commercial or financial relationships that could be construed as a potential conflict of interest.

Copyright (c) 2017 Viciani, Montagnani, Tordini, Romano, Salerni, De Luca, Ruggiero and Manetti. This is an open-access article distributed under the terms of the Creative Commons Attribution License (CC BY). The use, distribution or reproduction in other forums is permitted, provided the original author(s) or licensor are credited and that the original publication in this journal is cited, in accordance with accepted academic practice. No use, distribution or reproduction is permitted which does not comply with these terms. 